\title{
Geometric effects on fluid mixing in passive grooved micromixers
}

\author{
Jing-Tang Yang,* Ker-Jer Huang and Yu-Chun Lin \\ Received 20th January 2005, Accepted 6th June 2005 \\ First published as an Advance Article on the web 18th August 2005 \\ DOI: 10.1039/b500972c
}

The effects of geometric parameters on the mixing performance of a staggered herringbone mixer (SHM) with patterned grooves are numerically investigated. Combining use of the software package CFD-ACE+ and the Taguchi method provides a powerful and systematic approach for research on microfluidics. An orthogonal array $\mathrm{L}_{9}\left(3^{4}\right)$ is established for parameters introduced by the groove geometry; in total 9 cases are simulated. Analyses of the mixing phenomena, geometric parameter, pressure loss and flow rate through grooves are conducted. The modes of fluid motion and dominant mechanisms of mixing within the SHM are observed and ascertained. Although the depth ratio and the asymmetry index of the groove are found to be dominant geometric parameters, the rate of flow within the groove is verified to be the most significant factor that affects the mixing performance of a SHM. To date, the effects of the parameters are evaluated within specified ranges, and the true optimum design has yet to be discovered.

\section{Introduction}

Featuring compactness and ease of fabrication, passive micromixers are widely utilized in Lab-on-a-Chip systems and micro total analysis systems ( $\mu \mathrm{TAS}$ ). Generally, fluids within the passive micromixer are stretched and folded when passing the channel, which effectuates mixing. A micromixer with a three-dimensional serpentine channel was developed by Liu et $a{ }^{1}$ The serpentine flow passage kept twisting the interface between the two fluids, which produced chaotic advection and enhanced mixing at a higher Reynolds number.

Stroock et al. ${ }^{2-4}$ developed a novel version of the T-shaped micromixer; the staggered herringbones were patterned on the bottom wall, and the helical motions of the fluid driven by the grooves were observed to result in better mixing. Wang et al. ${ }^{5}$ conducted numerical investigations into this type of micromixer. Deeper grooves were found to improve the mixing efficiency and reduce the channel length required for complete mixing. The flow was also found ${ }^{6}$ to be stretched and folded by the patterned groove, so that the diffusion path was reduced noticeably and rapid mixing was made possible.

A slanted well was patterned at the bottom wall of the T-shaped channel by Johnson et al., ${ }^{7,8}$ which realized quick mixing by introducing transverse transportation of the fluids. Two counter-rotating vortices were found in the channel of the staggered herringbone mixer (SHM) by Aubin et al., ${ }^{9}$ in which the CFD software package CFX5 was adopted for simulations. Another numerical investigation (CFX 4.2) on the singlesided base-relief structured channel performed by Schönfeld and $\mathrm{Hardt}^{10}$ showed a consistent ratio of average velocities with that of the experiments of Stroock et al. ${ }^{3}$ On the other hand, a theoretical analysis utilizing a colored particle tracking method conducted by Kang et al. ${ }^{11}$ demonstrated consistent results with those of Stroock et al. ${ }^{3}$

Department of Power Mechanical Engineering, National Tsing Hua

University, Hsinchu, Taiwan 30013. E-mail: jtyang@pme.nthu.edu.tw
The T-shaped and Y-shaped micromixers are both fundamental microfluidics configurations. When the flow field is scaled down to behave in the microchannels, the surface to volume ratio increases drastically so that the boundary conditions become more dominant. Hence the staggered herringbone mixer (SHM) with patterned grooves is more effective at microscale due to the significant dominance of the surface effects. Although there have been many studies concerning the staggered herringbone mixer, a systematic investigation into geometric effects on the SHM's performance was found to be rare.

The Taguchi method was found to be a powerful tool in the research field of MEMS. ${ }^{12,13}$ By adopting this approach, the number of experimental parameters is reduced and the sensitivity of each parameter is analyzed. In this study, influences upon the SHM's performance of the geometric parameters that have not yet been studied are investigated numerically. The work is conducted in a more systematic manner by introducing the Taguchi method. The orthogonal array used here is $\mathrm{L}_{9}\left(3^{4}\right)$, so that the number of simulations required for a thorough analysis is reduced from $3^{4}=81$ to 9 . The grid independence is estimated at the very beginning; the sensitivity of the geometric parameters and the optimum design considering the mixing index are then obtained simultaneously.

\section{The orthogonal array}

Considering the geometric parameters, the intersection angle of the groove in the SHM developed by Stroock et al. ${ }^{2}$ was fixed to be $90^{\circ}$; an optimization study by Johnson and Locascio $^{7}$ showed that better mixing was achieved by increasing the depth and decreasing the inclination (angle formed between the groove and the channel) of the groove. Further, the influence of the number of subsections has been studied numerically by Jen et $a .^{14}$ The optimal mixing performance was obtained for a twisted micromixer with 6 subsections. 


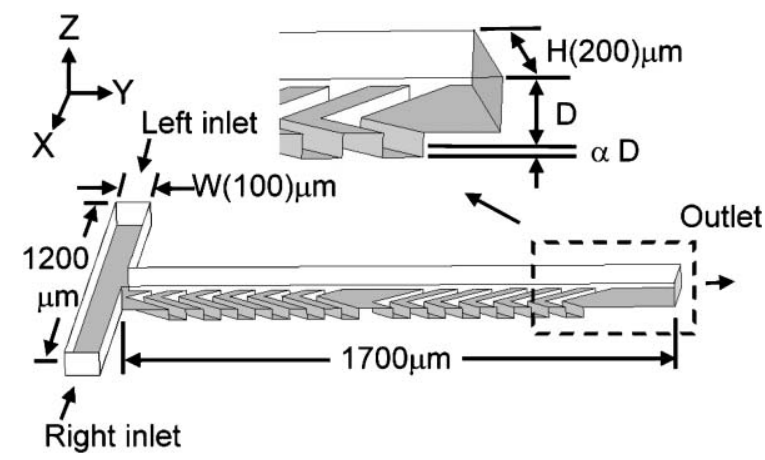

Fig. 1 The geometry of the staggered herringbone mixer (not to scale).

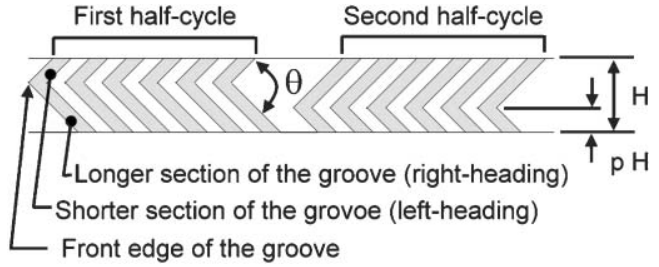

Fig. 2 Parameters related to the geometry of the groove.

Besides the factors mentioned above, there are other geometric parameters that have not yet been thoroughly studied. Since the performance of the SHM, or the quantity "mixing index," is considered to be dominated by the groovedriven helical motions of the fluid, the parameters relating to the groove geometry are focused upon in this investigation. These factors include the asymmetry index $p$, the depth ratio of the groove $\alpha$, the groove intersection angle $\theta$, and the $W / H$ ratio. The geometry of the whole SHM is shown in Fig. 1, where $\mathrm{D}-\alpha \mathrm{D}$ is equal to $70 \mu \mathrm{m}$, and the geometric parameters introduced by the groove are shown in Fig. 2.

Due to the complexity of the microfluidics experiment, computational fluid dynamics are utilized in this study for a shorter device design cycle and more physical insight into the mixing process is given. The numerical value of each parameter is listed in Table 1, and the established orthogonal array is shown as Table 2. The Reynolds number is set to be 10 and the Peclet number is 20000 in this study.

\section{Numerical simulations}

The governing equations of the isothermal incompressible steady-state mixing process in the staggered herringbone mixer are the continuity equation, the Navier-Stokes equations and the species convection-diffusion equations (gravity force is neglected).

Table 1 Numerical values of geometric parameters

\begin{tabular}{lllll}
\hline No. & Parameter & 1 & 2 & 3 \\
\hline A & Asymmetry index $(p)$ & 0.21 & 0.33 & 0.45 \\
B & Depth ratio of the groove $(\alpha)$ & 0.07 & 0.13 & 0.18 \\
C & Upstream to downstream channel & 0.5 & 1 & 1.5 \\
$\quad$ width ratio $(W / H)$ & & & \\
D & Groove intersection angle $(\theta)$ & $60^{\circ}$ & $90^{\circ}$ & $120^{\circ}$ \\
\hline
\end{tabular}

\section{Continuity equation}

$$
\frac{\partial u_{k}}{\partial x_{k}}=0
$$

Momentum equation

$$
u_{k} \frac{\partial u_{i}}{\partial x_{k}}=-\frac{1}{\rho} \frac{\partial p}{\partial x_{i}}+\frac{\mu}{\rho} \frac{\partial}{\partial x_{k}}\left(\frac{\partial}{\partial x_{k}} u_{i}\right)
$$

Species equation

$$
u_{k} \frac{\partial c}{\partial x_{k}}=D \frac{\partial}{\partial x_{k}}\left(\frac{\partial c}{\partial x_{k}}\right)
$$

The boundary conditions are

inlet: $u_{\mathrm{x}}=$ constant, $u_{\mathrm{y}}=0, u_{\mathrm{z}}=0, c=$ constant, $p=$ constant;

outlet: $\frac{\partial u_{i}}{\partial x_{y}}=0, \frac{\partial c}{\partial x_{y}}=0, p=0$

wall: $u_{\mathrm{i}}=0$ (no slip condition), where $u_{\mathrm{i}}$ : the velocity in the $\mathrm{i}$ direction

$p$ : pressure

$\rho$ : density of the fluid

$\mu$ : viscosity of the fluid

$D$ : molecular diffusivity

$c$ : species concentration.

The numerical simulations of the nine cases in Table 2 are conducted by utilizing the CFD software package CFD-ACE+ of CFD Research Corp. The SIMPLEC method is adopted for pressure-velocity coupling. The central differencing scheme (with blending set to 0.1 ) and second-order upwind scheme are adopted for the velocity and concentration calculations, respectively ${ }^{15}$ (CFD-ACE 2002 user manual). The coefficients of viscosity and diffusion are set to be constant. No chemical reaction is considered to happen between the fluids entering from the two inlets. The concentration of the species is normalized to be one for the inlet in the positive- $x$ direction (right) and zero for the opposite inlet (left). The solution is considered to be converged when the following criterion has been met for all dependent variables between the sweeps $n$ and $n+1$ :

$$
\max \left|\frac{\phi^{n+1}-\phi^{n}}{\phi_{r}}\right| \leq 10^{-4}
$$

Note that $\phi_{\mathrm{r}}$ represents the reference value of the dependent variable $\phi$.

The present simulations are performed on a highly variable mesh, as shown in Fig. 3. The mesh is constructed to capture not only the large gradients of concentration but also the

Table $2 \quad \mathrm{~L}_{9}\left(3^{4}\right)$ orthogonal array

\begin{tabular}{lllll}
\hline Case no. & A & B & C & D \\
\hline 1 & 1 & 1 & 1 & 1 \\
2 & 1 & 2 & 2 & 2 \\
3 & 1 & 3 & 3 & 3 \\
4 & 2 & 1 & 2 & 3 \\
5 & 2 & 2 & 3 & 1 \\
6 & 2 & 3 & 1 & 2 \\
7 & 3 & 1 & 3 & 2 \\
8 & 3 & 2 & 1 & 3 \\
9 & 3 & 3 & 2 & 1 \\
\hline
\end{tabular}




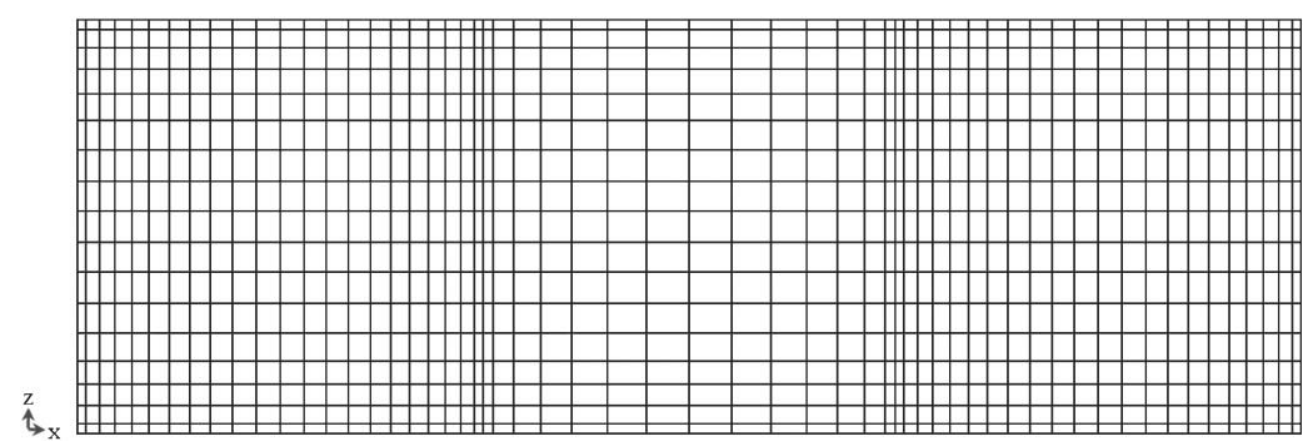

Fig. 3 Mesh arrangement of the staggered herringbone mixer channel at the outlet.

boundary layers near the fluid-solid interface. Also, the mesh is arranged to be capable of providing sufficient resolution at the grooved surfaces with minimum element distortion.

An analysis of the grid size sensitivity has been conducted at the very beginning. The latter case has been selected for further investigation such that the overall mixing index variation does not exceed $8 \%$ in the micromixer (Table 3). In Fig. 4, the simulated concentration distribution is in agreement qualitatively with the previously published experimental result. ${ }^{2}$

\section{Mixing phenomena}

By inspecting the results from the numerical simulations, the fundamental mixing phenomena within the SHM are clarified. If there are no grooves present, the concentration distribution at the outlet is anticipated to possess a similar pattern to that at position $Y=0$. However, a comparison between the concentration distributions at the cross-section $Y=0$ and the outlet $Y=1.7 \mathrm{~mm}$ (Fig. 5) of the SHM reveals an evident difference. The bulk advection is then considered to be significant during the mixing process. The whole mixing effects in the SHM are considered to be the combined results of molecular diffusion between the two fluids and the bulk advection caused by the grooves at the bottom wall.

The streaklines (Lagrangian trajectories) of the flow within the SHM (Fig. 6a) reveal more details about the bulk advection. The fluid from the left inlet (in the negative- $x$

Table 3 Grid sensitivity analysis

\begin{tabular}{lllll}
\hline $\begin{array}{l}\text { Number } \\
\text { of nodes }\end{array}$ & $\begin{array}{l}\text { Pressure } \\
\text { loss } / \mathrm{kg} \mathrm{m}^{-2}\end{array}$ & $\begin{array}{l}\text { Average } \\
\text { mixing } \\
\text { index }\end{array}$ & $\begin{array}{l}\text { Relative } \\
\text { difference } \\
\text { in pressure (\%) }\end{array}$ & $\begin{array}{l}\text { Relative } \\
\text { difference } \\
\text { in mixing } \\
\text { index (\%) }\end{array}$ \\
\hline 37378 & 407.0 & 0.18 & - & - \\
455184 & 429.9 & 0.14 & 5.3 & 28.6 \\
900612 & 427.6 & 0.13 & 0.5 & 7.6 \\
\hline
\end{tabular}

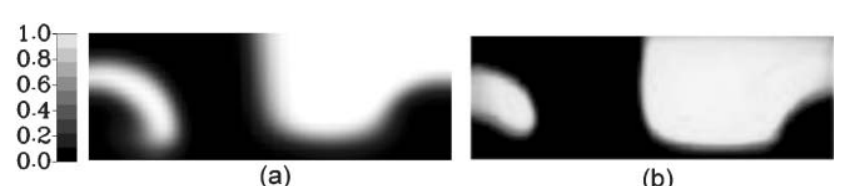

Fig. 4 Comparison between (a) the simulated concentration distribution with (b) the previously published result ${ }^{2}$ for $\mathrm{Pe}=20000$. direction) first reaches the front edge of the groove. It is then divided into two streams. The stream closer to the left side of the groove front edge enters the shorter left-heading section of the groove, goes beneath the flow from the right inlet (in the positive- $x$ direction) in the groove channel, rolls out from the groove at the side edge, and finally goes back to the mainstream (Fig. 6b). The action results in the clockwise rotation of the flow in the mixing channel.

The other stream enters the longer right-heading section of the groove, goes beneath the flow from the right inlet in the groove channel, rolls out from the groove at the side edge, and finally goes back to the mainstream (Fig. 6c). The action results in the counter-clockwise rotation of the flow. Besides, a small scale helical motion is observed (Fig. 6d), which is similar to the result from Wang et al. ${ }^{5}$ However, because of the similar size of the grid and the helical scale, this flow mode has yet to be further clarified. Note that minor helical motions of the flow are also observed at the interface between the groove and the mixing channel as part of the flow from the left inlet ripples over.

As far as the fluid coming from the right inlet is concerned, while some of it flows downstream directly above the groove, the rest is either trapped into the groove or ripples over the groove. It is believed that the second and third modes of flow are beneficial to the effects of mixing, while there is almost no contribution by the first flow mode.

Contributions to mass transfer and mixing by bulk advection of the flow are more evidently demonstrated in Fig. 7. As the flow goes downstream, the fluid coming from the left inlet is guided beneath the flow coming from the right inlet. The groove-guided motions of the flow result in increased contact area between the two fluids and the enlarged velocity vectors vertical to that of the mainstream in the mixing channel, which both enhance the degree of mixing. In Fig. 7, there is a mixed zone at the lower-right side due to the flow rolling back from the longer right-heading groove section.

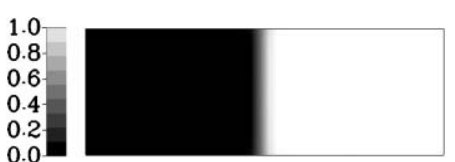

(a) $\mathrm{Y}=0$

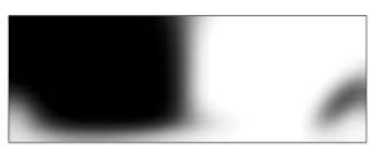

(b) $Y=1.7 \mathrm{~mm}$
Fig. 5 Concentration distributions at the two cross-sections (a) $Y=0$ and (b) $Y=1.7 \mathrm{~mm}$. 


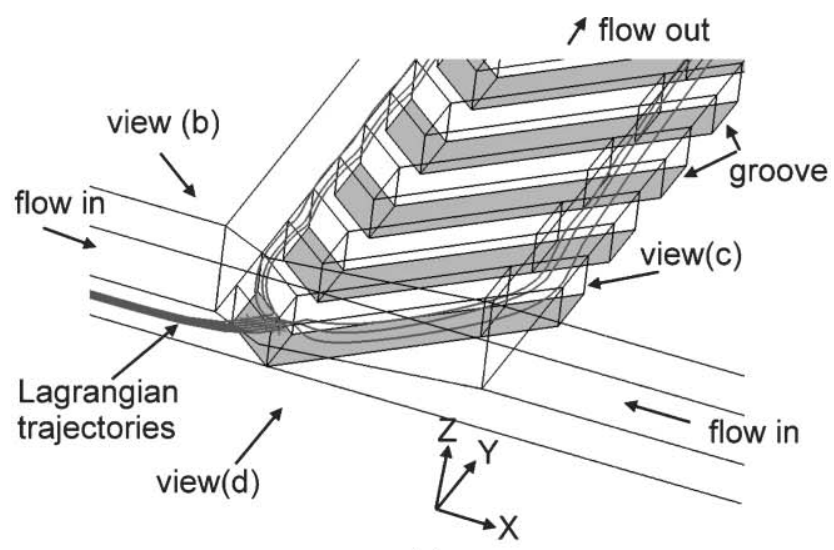

(a)

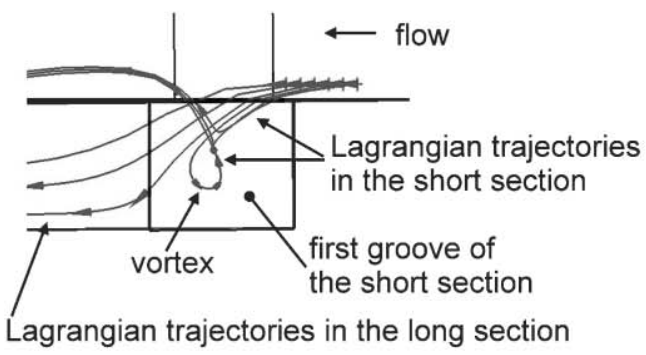

(b)

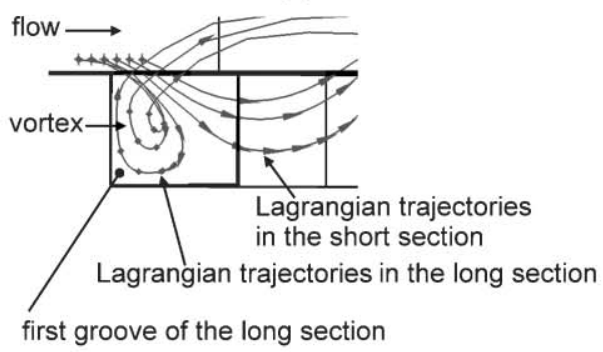

(c)

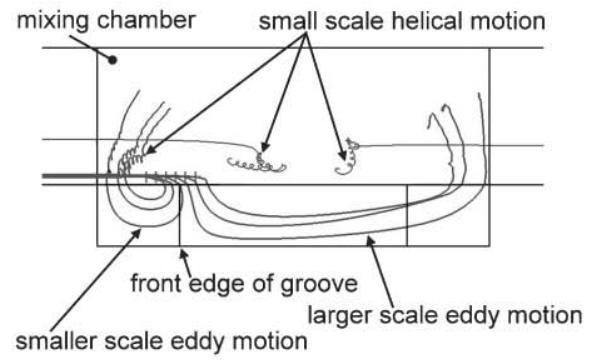

(d) The streakline-projection on the X-Z plane

Fig. 6 Lagrangian trajectories of the flow within the staggered herringbone mixer.

A similar situation happens at which the headings of the longer and the shorter groove sections are changed to the opposite directions. The fluid in the right, which has been mixed to a certain extent at the prior stage, starts being transmitted to the left by the longer left-heading groove section. Since the fluids within the mixing channel are first turned clockwise and then counter-clockwise by the flow rolling back from the grooves, the final interface between the two fluids remains in the middle of the channel. But two zones with uniformly-distributed

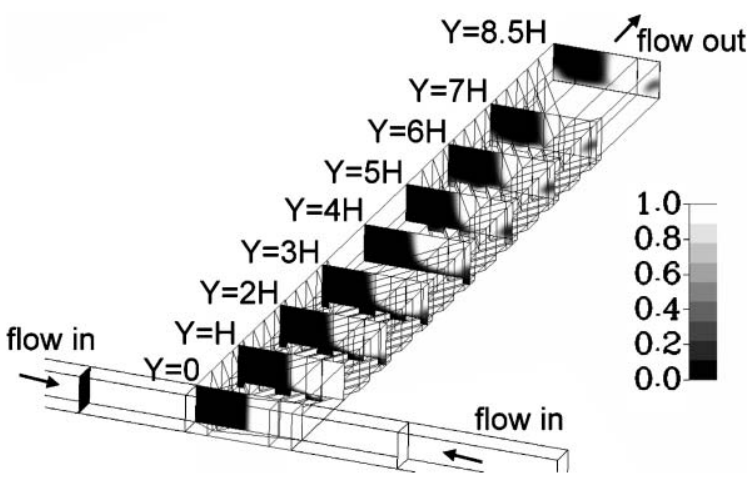

Fig. 7 Simulated concentration distributions at various cross sections from Case 1 .

concentration are also generated at the lower-right and lowerleft side, respectively. The contribution to mixing by the rolling-back of the flow from the grooves is then verified by the coincidence between the locations of the well-mixed zones and those of the vortical flow motions as seen in Fig. 7.

According to the above analysis, at lease two mechanisms are considered to dominate the mixing process within the SHM. While the vortical motions of the flow at the side edge result in the stretching and folding of the interface between the two fluids, which was proposed by Stroock et al. ${ }^{2}$ and Schönfeld and Hardt, ${ }^{10}$ the underside fluid transportation by the grooves is believed to increase the contact area between the two fluids. In other words, fluid transportation within the groove induces large vortical and minor helical motions within the mixing channel. These motions result in an increase of the contact area between the two fluids and the flow vertical to the mainstream direction, which both enhance mixing. It is then reasonable to expect that the flow rate within the groove is one of the factors dominating the mixing performance of the SHM. The significant role played by the grooves in the SHM will be further discussed in the next section.

\section{Geometric parameter analysis}

Based on the results from the simulations, the square of standard deviation of the concentration at the end plane (outlet) is calculated by eqn. (5) for each case. The mixing index (Mi) and the signal to noise ratio $(\eta)$ are also evaluated by eqns. (6) and (7), respectively (Table 4). Better mixing

Table 4 The mixing index, square of standard deviation and signal to noise ratio of the orthogonal array in Table 2

\begin{tabular}{llll}
\hline Case no. & $\begin{array}{l}\text { Mixing } \\
\text { index }(\mathrm{Mi})\end{array}$ & $\begin{array}{l}\text { Square of standard } \\
\text { deviation }\left(\sigma^{2}\right)\end{array}$ & $\mathrm{S} / \mathrm{N}(\eta)$ \\
\hline 1 & 0.11 & 0.20 & 7.02 \\
2 & 0.14 & 0.18 & 7.33 \\
3 & 0.14 & 0.18 & 7.37 \\
4 & 0.10 & 0.20 & 6.93 \\
5 & 0.12 & 0.19 & 7.10 \\
6 & 0.15 & 0.18 & 7.41 \\
7 & 0.10 & 0.20 & 6.94 \\
8 & 0.11 & 0.20 & 7.07 \\
9 & 0.10 & 0.20 & 6.93 \\
\hline
\end{tabular}


Table 5 The influence of each parameter on the signal to noise ratio

\begin{tabular}{lllll}
\hline & $\eta_{\mathrm{A}}$ & $\eta_{\mathrm{B}}$ & $\eta_{\mathrm{C}}$ & $\eta_{\mathrm{D}}$ \\
\hline 1 & 7.24 & 6.96 & 7.16 & 7.02 \\
2 & 7.15 & 7.17 & 7.07 & 7.23 \\
3 & 6.98 & 7.23 & 7.14 & 7.12 \\
Max-min & 0.26 & 0.27 & 0.10 & 0.21 \\
\hline
\end{tabular}

performance is revealed by the larger values of either the mixing index or the signal to noise ratio.

$$
\begin{gathered}
\sigma^{2}=\frac{1}{n} \sum_{i=1}^{n}\left(c_{i}-c_{\infty}\right)^{2} \\
\mathrm{Mi}=\left(1-\frac{\int_{A}\left|c_{i}-c_{\infty}\right| \mathrm{d} A}{\int_{A}\left|c_{0}-c_{\infty}\right| \mathrm{d} A}\right) \\
\eta=\frac{S}{N}=-10 \log \sigma^{2}
\end{gathered}
$$

where $c_{i}$ is the concentration distribution at the end plane of the $i^{\text {th }}$ species, $c_{0}$ is the initial concentration at the inlet, $c_{\infty}$ is the concentration of complete mixing, $\mathrm{Mi}$ is the mixing index, $\sigma^{2}$ is the square of standard deviation, and $\eta$ is the signal to noise ratio.

To evaluate the mixing performance of the SHM, a static Taguchi analysis for the problem with the character of "the smaller the better" has been performed. Investigating further the results from Table 4, the influence of each parameter on the signal to noise ratio is demonstrated in Table 5 .

The results from Table 5 are depicted in Fig. 8. The positive slope of the curve in Fig. 8 indicates that increasing the corresponding parameter results in a higher mixing index, and vice versa. The mixing performance is found to be most sensitive to the depth ratio of the groove $\alpha$. The relative effectiveness of the geometric parameters is ranked as:

$$
\alpha \sim p>\theta>W / H
$$

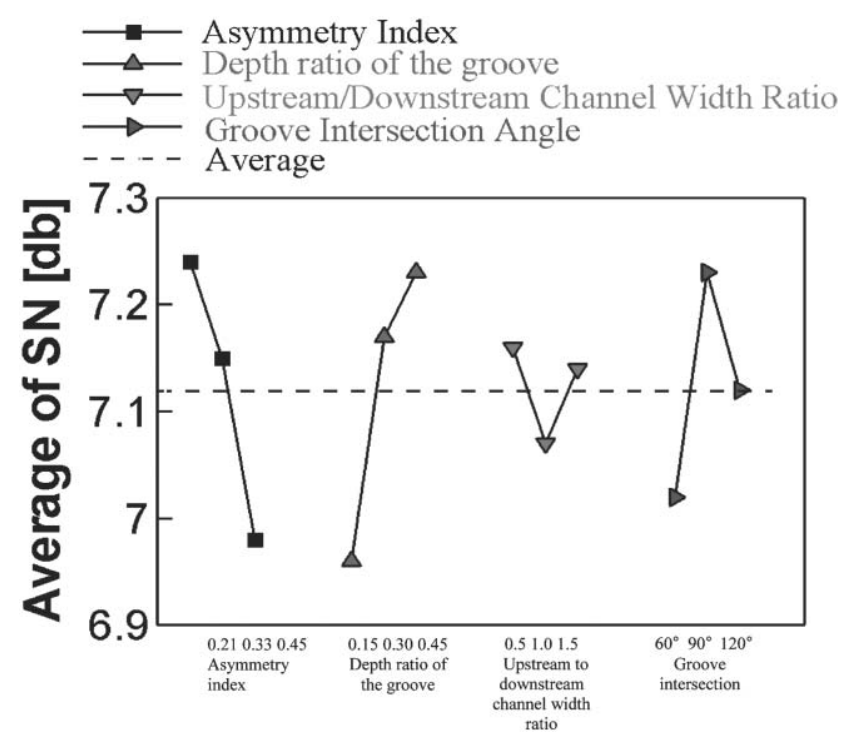

Fig. 8 Influence of design parameters on signal to noise ratio.

\section{The depth ratio of the groove $\alpha$}

The depth ratio of the groove is found to be one of the most effective parameters in this analysis. According to the flow simulation, it is evident that the flow rate increases as the groove becomes deeper. Also, the large vortical motions of the fluid at the side edge of the groove are enhanced by the increased groove depth, so that the mixing is significantly improved as the depth of the groove increases. This result is consistent with Johnson and Locascio's work. ${ }^{7}$ The connection between the flow rate within the groove and the mixing index is further verified in the following sections.

\section{The asymmetry index $p$}

The longer section of the asymmetric-V groove in the SHM is believed to be capable of transporting the fluid to another side of the channel which was originally occupied by the other fluid. So, asymmetry is favored for better mixing due to the more effective transportation capability. If the front edge of the groove is close to the center plane $(X=0)$ of the mixing channel, there is less substantial transportation of the fluid to the other side of the channel. Thus the improvement on mixing is poor. From another perspective, since the scales of the two vortices within the mixing channel are actually determined by the asymmetry index, two vortices with dissimilar scales are demonstrated to provide a better mixing performance than two equally-sized ones. According to Schönfeld and Hardt, ${ }^{10}$ chaotic advection was introduced by alteration of the flow motion; and this further verifies the more effective mixing by asymmetric vortices. However, the result doesn't imply that the best mixing performance is achieved when the asymmetry index is equal to zero; no chaotic advection is introduced in this case where only one vortex is produced.

\section{The groove intersection angle $\theta$}

There is only a mild impact on mixing by changing the groove intersection angle. Though this result is not explicitly demonstrated by the flow field within the SHM, the effect of the intersection angle is further clarified when considering the pressure loss within the grooves.

\section{The upstream to downstream channel width ratio $\mathrm{WIH}$}

Since the effect of the width ratio is restricted to redistribute the two fluids in the intersection of the inlets and the mixing channel, there is no significant impact on the overall mixing performance of the SHM. A more substantial effect by this parameter is expected for cases with high Reynolds numbers.

After clarifying the effect of each parameter on mixing, the optimum design of the SHM is also expected to be discovered. The optimum geometry obtained from Table 5 is $A_{1} B_{3} C_{1} D_{2}$, and the corresponding signal to noise ratio for the optimum design according to the Taguchi method is evaluated as:

$$
\begin{gathered}
\eta_{\text {optimum }}=A_{1}+B_{3}+C_{1}+D_{2}-3 \times T=7.24+7.23+7.16+ \\
7.23-3 \times 7.12=7.50
\end{gathered}
$$

where $T=$ average signal to noise ratio of all cases. 


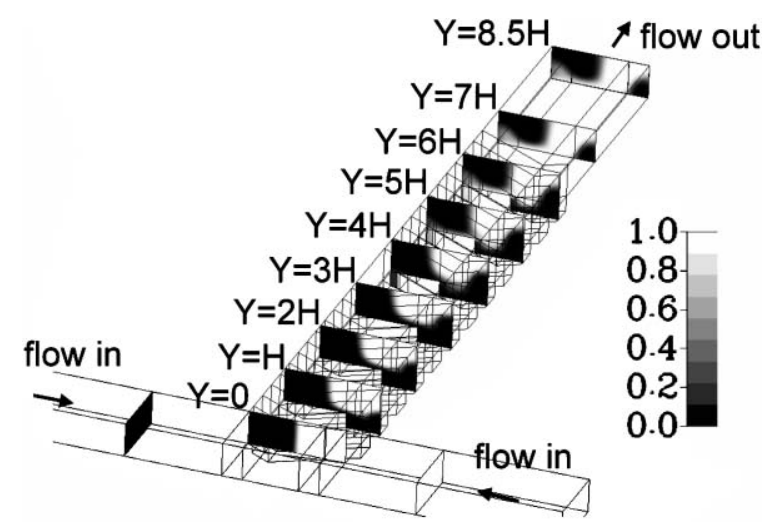

Fig. 9 Simulated concentration distributions at various cross sections from Case 3.

The optimum geometry is examined again by performing simulations on CFDRC-ACE+. The results show that the square of standard deviation $\sigma^{2}$ is 0.19 , which implies that the signal to noise ratio $\eta$ is only 7.22. The deviation from the result of the Taguchi method is considered to be caused by crosstalk effects among the selected geometric parameters, which means that these parameters are not independent from each other. Though the Taguchi method is capable of providing a thorough analysis of the geometric parameters, it is still limited by the selection of the parameters. So, according to the results from the nine simulated cases, the optimum design in this study is considered to be that of Case 6 .

Since parameters $\alpha$ and $p$ are considered to be dominant, the major difference between Cases 1 and 3 is the depth of the groove. The concentration distributions at several cross sections within the SHM in Case 3 are shown in Fig. 9. It is evident that the mixing performance in Case 3 is much better than that in Case 1. The reason for that is considered to be the deeper groove in Case 3, which is capable of transporting more fluid for mixing. The vortical motions of the fluid induced by the groove are also enhanced, which is demonstrated by the large well-mixed zone at the lower-right side of Fig. 9.

\section{Pressure loss analysis}

As far as the micromixer is concerned, pressure loss through the mixing channel is critical to its practicability and must be evaluated during the design phase. Pressure loss across the SHM for all cases are plotted in Fig. 10. No significant connection between pressure loss and the mixing index is observed. The higher pressure loss is considered to be a consequence of the smaller width of the inlet channel. Such as in Cases 1,6 and 8, the channel width of the inlet is only $50 \mu \mathrm{m}$ and the pressure loss achieves a maximum of $1000 \mathrm{~Pa}$. No substantial effects on the pressure loss are observed for the other three parameters ( $p, \alpha$ and $\theta$ ). A SHM with good mixing performance and low pressure loss is then considered to be realizable.

However, since the flow rate within the groove is expected to be the dominant factor affecting mixing performance, we focused on pressure loss within the groove instead. Based on the uniform pressure distribution at each $\mathrm{X}-\mathrm{Z}$ plane in the

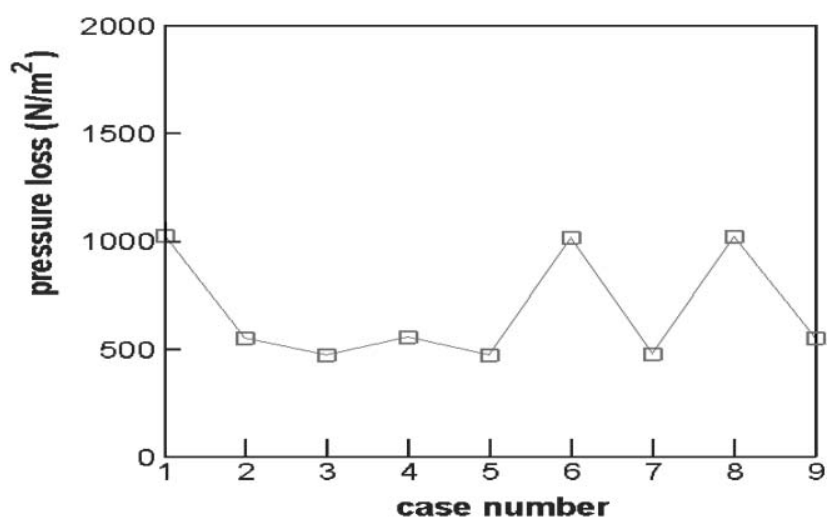

Fig. 10 Pressure loss across the SHM.

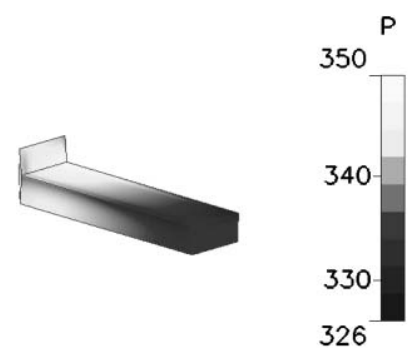

Fig. 11 Pressure distribution within a groove.

mixing channel, the pressure at the front edge of the groove is always larger than that at the rear end. The pressure generally decreases along the side wall of the groove (Fig. 11). So the fluid is pushed into the groove and results in enhanced mixing as discussed before.

The relationships between pressure loss along the groove and the geometric parameters are considered to be relevant to the flow rate within the groove. Since one of the groove sections shrinks as parameter $p$ decreases, the flow rate is increased due to the enlarged pressure difference along the longer section of the groove. On the other hand, increasing parameter $\alpha$ actually increases the cross-sectional area of the groove, thus the flow resistance is reduced and the flow rate is raised. The trend is consistent with the results from the Taguchi method, that is, decreasing the asymmetry index $p$ or increasing the depth ratio of the groove $\alpha$ both increase the flow rate within the groove and uplift the mixing performance.

The effect of parameter $\theta$ on the flow rate is not explicit. While increasing $\theta$ reduces both pressure difference and flow resistance, decreasing it actually results in a larger pressure difference and flow resistance. As a result of the offsets, the impact on mixing by parameter $\theta$ is less significant than parameters $p$ and $\alpha$. The character is again in agreement with analysis based on the Taguchi method.

\section{Groove flow rate analysis}

According to previous analyses (flow field, geometric parameters, pressure loss), the flow rate in the groove is one of the most important factors dominating the mixing performance of the SHM. To further clarify this connection, the flow rate within the grooves of the SHM is directly evaluated by 


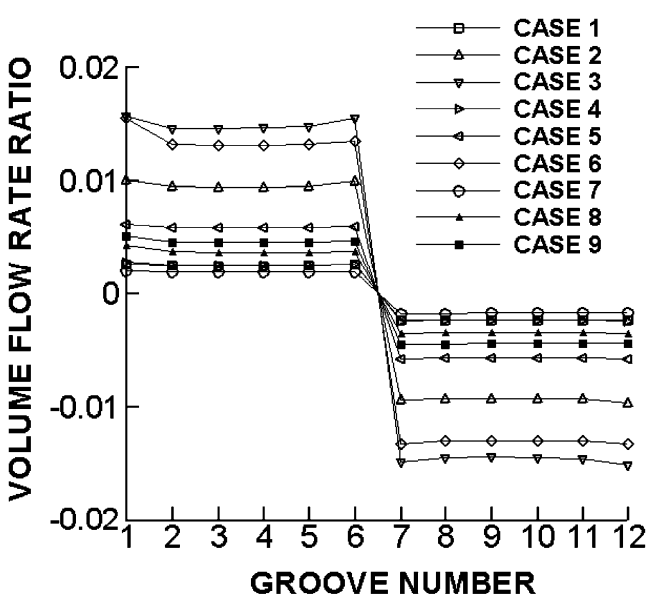

Fig. 12 Volume flow rate ratio of each groove.

integrating the velocity field over the center plane $(X=0)$ in the mixing channel. A total of twelve grooves are divided into two mixing half-cycles; while the longer section of the groove is left-heading in the first half-cycle (groove numbers 1-6), it orients to the right in the second half-cycle (groove numbers 7-12). The total groove flow rate in the first halfcycle is considered as the "positive" flow rate, and that of the second half-cycle is viewed as a "negative" value. For the nine simulated cases, the ratio of the flow rate in each single groove to the total flow rate within the mixing channel is demonstrated in Fig. 12. The ratio is in the range from 0.18 to $1.56 \%$.

It is noted that the volume flow rate ratio of the first groove is the maximum among all the grooves. Also, the flow rate ratios of the first and last grooves within each half-cycle are larger than those of the other grooves. The descending of the flow rate ratio is actually caused by "interference" among the grooves. The cross-sectional area is increased due to the existence of the groove, and the flow rate ratio of the groove is reduced as a result of the decreased pressure drop through the groove. Since the direction of the grooves changes as the fluids travel downstream to another half-cycle, it is evident that the flow rates of the first and last grooves in each half-cycle are less affected by pressure reduction than the other grooves. It should also be noted that while the fluid transported to the right in the first half-cycle is basically "pure," the groovedelivered fluid in the second half-cycle has been pre-mixed to a certain extent. This is demonstrated in both Fig. 7 and Fig. 9, in which the fluid is arriving at the leading edge of the second half-cycle. And, it is obvious that the fluid transported by the grooves then is already partially mixed.

A comparison among the mixing index, the sum of the groove volume flow rate ratio within the first half-cycle $\left(Q_{\mathrm{g}} / Q_{\mathrm{m}}\right)$, and the ratio of the total groove volume in the first half-cycle $\left(V_{\mathrm{g}}\right)$ to the total volume of the mixing channel $\left(V_{\mathrm{m}}\right)$ for the nine simulated cases is shown in Table 6 . Though there is only a three-times difference for $V_{\mathrm{g}} / V_{\mathrm{m}}$, the resulting difference for $Q_{\mathrm{g}} / Q_{\mathrm{m}}$ is as large as eight-times. Considering the top four rankings of the mixing index and the volume flow rate ratio $Q_{\mathrm{g}} / Q_{\mathrm{m}}$, it is found that the two rankings completely agree with each other. The coincidence evidently verifies a
Table 6 Comparison among the mixing index, the groove flow rate ratio, and the groove volume ratio

\begin{tabular}{llll}
\hline Case no. & $\mathrm{Mi}$ & $Q_{\mathrm{g}} / Q_{\mathrm{m}}(\%)$ & $V_{\mathrm{g}} / V_{\mathrm{m}}$ \\
\hline 1 & 0.11 & 1.5 & 0.05 \\
2 & 0.14 & 5.7 & 0.11 \\
3 & 0.14 & 8.9 & 0.16 \\
4 & 0.10 & 1.5 & 0.05 \\
5 & 0.12 & 3.5 & 0.11 \\
6 & 0.15 & 8.1 & 0.16 \\
7 & 0.10 & 1.1 & 0.05 \\
8 & 0.11 & 2.2 & 0.11 \\
9 & 0.10 & 2.8 & 0.16 \\
\hline
\end{tabular}

direct connection between the mixing index of the SHM and the groove volume flow rate ratio. And the flow rate within the grooves of the SHM is substantiated as the most critical geometric parameter. The significance of the other parameters is revealed only if the flow rates within the groove between two micromixers are close.

It is worth noticing that the volume occupied by the grooves is critical to the design concept of reducing the "dead volume" for a biochemical or biomedical device. Although the groove volume ratio in the first half-cycle is as much as $16 \%$, the groove flow rate ratio achieves merely $8.9 \%$ in the optimized case (Case 3). Other appropriate parameters must be sought for practical and optimum design as far as the biochemical or biomedical applications are concerned.

\section{Conclusions}

The effects of geometric parameters on the mixing performance of the staggered herringbone mixer (SHM) are numerically investigated in a systematic manner. The computational fluid dynamics software package CFD-ACE+ is utilized in combination with the Taguchi method. Four parameters introduced by the geometry of the patterned grooves are focused upon. A $\mathrm{L}_{9}\left(3^{4}\right)$ orthogonal array is established to reduce the number of simulations required for complete analysis. A total of nine cases $(\mathrm{Re}=10, \mathrm{Pe}=20000)$ are evaluated by a mixing phenomena study, geometric parameter analysis, pressure loss analysis, and groove flow rate analysis.

Three different modes of fluid motion are observed in the mixing phenomena study. The fluid either goes into the groove, or flows directly downstream. The motion modes result in at least two dominant mechanisms of mixing in the SHM. While the vortical motions of flow at the groove's side edge result in the stretching and folding of the interface between the two fluids, the underside fluid transportation by the grooves increases the contact area between the two fluids. Since the groove is involved with all of the substantial mixing mechanisms, the flow rate within the groove is then expected to play one of the most significant roles considering the mixing performance of the SHM.

Geometric parameter analysis, based on both the simulation results and the Taguchi method, reveal the relative effectiveness as: depth ratio of the groove $\sim$ asymmetry index $>$ groove intersection angle $>$ upstream to downstream channel width ratio. Only the first two parameters are considered to be 
essential. While both of them are directly relevant to the flow rate within the groove, the results from pressure loss analysis also support the possible significance of the groove flow rate. At last, the total flow rate within the grooves in the first mixing half-cycle is directly evaluated for each simulation case. The ranking of the groove flow rate and that of the mixing index are in agreement in the top four cases. The groove flow rate is verified as the most important factor affecting the mixing performance of the SHM.

\section{Acknowledgements}

This work was partially supported by the National Science Council of the Republic of China under contracts NSC 932623-7-032-053 and NSC 93-2623-7-032-054.

\section{References}

1 R. H. Liu, M. A. Stremler, K. V. Sharp, M. G. Olsen, J. G. Santiago, R. J. Adrian, H. Aref and D. J. Beebe, Passive mixing in a three-dimensional serpentine microchannel, J. Microelectromech. Syst., 2000, 9, 190.

2 A. D. Stroock, S. K. Dertinger, A. Ajdari, I. Mezic, H. A. Stone and G. M. Whitesides, Chaotic mixer for microchannels, Science, 2002, 295, 647.

3 A. D. Stroock, S. K. Dertinger, G. M. Whitesides and A. Ajdari, Patterning flows using grooved surfaces, Anal. Chem., 2002, 74, 5306.
4 A. D. Stroock, K. W. Stephan, A. Ajdari, I. Mezic, H. A. Stone and G. M. Whitesides, Laminar mixing apparatus and methods, Pat. no. WO 03/011443.

5 H. Wang, P. Iovenitti, E. Harvey and S. Masood, Numerical investigation of mixing in microchannels with patterned grooves, J. Micromech. Microeng., 2003, 13, 801.

6 H. Wang, P. Iovenitti, E. Harvey and S. Masood, Passive mixing in microchannels by applying geometric variations, Proc. SPIE-Int. Soc. Opt. Eng., 2003, 4982, 282.

7 T. J. Johnson and L. E. Locascio, Characterization and optimization of slanted well designs for microfluidic mixing under electroosmotic flow, Lab Chip, 2002, 2, 135

8 T. J. Johnson, D. Ross and L. E. Locascio, Rapid microfluidic mixing, Anal. Chem., 2002, 74, 45.

9 J. Aubin, D. F. Fletcher, J. Bertrand and C. Xuereb, Characterization of the mixing quality in micromixers, Chem. Eng. Technol., 2003, 26, 1262.

10 F. Schönfeld and S. Hardt, Simulation of helical flows in microchannels, AIChE J., 2004, 50, 771.

11 T. G. Kang and T. H. Kwon, Colored particle tracking method for mixing analysis of chaotic micromixers, J. Micromech. Microeng., 2004, 14, 891.

12 J. S. Go and Y. H. Cho, Experimental evaluation of anodic bonding process based on the Taguchi analysis of interfacial fracture toughness, Sens. Actuators, 1999, 73, 52.

13 J. J. Tsaur, C. H. Du and C. Lee, Investigation of TMAH for front-side bulk micromachining process from manufacturing aspect, Sens. Actuators, 2001, 92, 52.

14 C. P. Jen, C. Y. Wu, Y. C. Lin and C. Y. Wu, Design and simulation of the micromixer with chaotic advection in twisted microchannels, Lab Chip, 2003, 3, 77.

15 CFD-ACE user manual, version 2002, CFD Research Corporation. 